\section{A difficult case of Hodgkin Lymphoma with differential diagnosis of tuberculosis and sarcoidosis}

\author{
Nilüfer Göknar, Erkan Çakır, \\ Fatma Betül Çakır, Ozgur Kasapcopur, \\ Gulcin Yegen, Ahmet Hakan Gedik, \\ Faruk Oktem
}

Bezmialem Vakif University, Istambul, Turkey

\section{Abstract}

We report here the case of a 14-year-old boy with history of fever, weight loss, and mediastinal lymphadenopathy. The clinical symptoms and laboratory findings mimicking tuberculosis and sarcoidosis complicated the diagnostic process. He was diagnosed with Hodgkin's lymphoma after several X-rays, computed tomography, positron emission tomography-computed tomography, laboratory tests and three lymph node biopsy. Clinicians should be alerted on new lesions and symptoms in high risk patients and should repeat diagnostic tests and lymph node biopsies as indicated.

\section{Introduction}

Hodgkin's lymphoma (HL) is a curable malignancy which most patients present with superficial asymptomatic lymphadenopathy. Cervical, supraclavicular and mediastinal lymph nodes are the most common involvement sites. An enlargement in mediastinum is a frequent found on chest X-rays., ${ }^{1,2}$ The Epstein-Barr virus (EBV) which is an oncogenic herpes virus has been implicated in the pathogenesis of HL. ${ }^{3}$ In economically developing countries, HL displays a bimodal curve. But in economically underdeveloped countries the overall incidence is lower except adolescence and young adulthood. ${ }^{1,4}$

We report a difficult case of a boy with HL mimicking tuberculosis and sarcoidosis. Because of the similarity of clinical findings, the diagnosis could be done eight months after the onset of symptoms.

\section{Case Report}

A fourteen-year-old boy presented with weight loss, myalgia, depressed appetite, and leg pain and was referred to our pulmonology unite to check out the possibility that he might have tuberculosis. Physical examination revealed cervical lymphadenomegaly, erythema nodosum, and arthritis (Figure 1 for the timeline). White blood cell (WBC) count, hemoglobin $(\mathrm{Hb})$, and erythrocyte sedimentation rate (ESR) were $18,000 / \mathrm{mm}^{3}, 12 \mathrm{~g} / \mathrm{dL}$, and $30 \mathrm{~mm} /$ hour, respectively. A thorax computed tomography (CT) scan showed mediastinal extensive lymphadenomegaly. Screening of other members of his family was negative for tuberculosis. The patient had one Bacillus Calmette-Guérin scar. The tuberculin skin test, tuberculosis bacilli examinations of gastric aspirate, and conventional cultures were performed with negative results. Bronchoscopy revealed an appearance consistent with an external compression on the the main lobe of the right lung. Bronchoalveolar lavage was taken during bronchoscopy for microbiological investigations and results were again negative for tuberculosis. After the initial testing and upon recommendations of his relatives, the patient was prescribed prednisone treatment for two weeks because of severe pain. Afterwards lymph node biopsy was done by mediastinoscopy and revealed CD4 dominant T lymphocyte-rich lymphoid cell proliferation with scanty atypical cells looking suspiciously similar to Reed-Stenberg cells. Atypical cells were positive for CD30, some of which were also positive for EBV-I3 and fascin. CD15 was negative. Immunohistochemistry and the morphology did not fulfill the diagnostic criteria of Hodgkin's lymphoma. Granuloma was absent on pathological investigation of biopsy to conform either tuberculosis or sarcoidosis. As a result of all these findings, the pathologic investigation showed a possible chronic EBV infection or EBV-induced lymphoproliferative disease. EBV VCA IgM, IgG, and EBV DNA copy were negative, positive, and 938, respectively. These results suggested a possible chronic EBV infection. Non-steroidal anti-inflammatory drugs were prescribed. After one month, the patient was hospitalized for his persistent severe pain, arthritis in his right foot, and cervical lymphadenomegaly. As his blood pressure was $150 / 100 \mathrm{mmHg}$, enalapril treatment was started. Eye examination revealed uveitis sequelae for which the patient had been treated four months prior. Laboratory examination


platelets: $612 \times 10^{3} / \mu \mathrm{L}, \mathrm{LDH}: 225 \mathrm{U} / \mathrm{L}$, and ESR: $44 \mathrm{~mm} / \mathrm{hr}$. Thorax CT showed bilateral axillary multiple lymph nodes with a dimension of 20 $\mathrm{mm}$, mediastinal and hiler subcarinal packs of lymph nodes $(47 \times 27 \mathrm{~mm})$, a round $47 \times 37 \times 30$ $\mathrm{mm}$ lesion on the middle lobe of the right lung, and bilateral minimal pleural effusion. Bone marrow examination was normocellular. Because of the suspicion of lymphoma, a 18Fluoro-deoxyglucose (FDG) CT was performed (Figure 2). Values ranged from 2.3 to 8.7 , con-
Correspondence: Nilüfer Göknar, Bezmiâlem Vakıf Üniversitesi, Adnan Menderes Bulvarı (Vatan Caddesi), Fatih, İstanbul, Turkey

Tel.: +90.0212.5232.288 - Fax: +90.0212 .4531 .870 .

E-mail: nilufergoknar@gmail.com

Key words: Sarcoidosis; lymphoma; tuberculosis; Behçet's disease.

Acknowledgements: the authors appreciate the contributions and editorial assistance made by $\mathrm{S}$. Delacroix

Contributions: the authors contributed equally.

Conflict of interest: the authors declare no potential conflict of interest.

Received for publication: 20 September 2014. Accepted for publication: 24 February 2015.

This work is licensed under a Creative Commons Attribution NonCommercial 3.0 License (CC BYNC 3.0).

CC Copyright N. Göknar et al., 2015

Licensee PAGEPress, Italy

Hematology Reports 2015; 7:5644

doi:10.4081/hr.2015.5644

sistent with the presence of inflammation. Cervical lymph node biopsy was done and lymphoma was excluded with this biopsy and FDG PET-CT. Also granuloma was absent in the biopsy.

The next step was to investigate the patient for sarcoidosis. The angiotensin-converting enzyme (ACE) level was 91 U/L. He also had considerable hypercalciuria and a low parathormone level. Pulmonary function test was compatible with restrictive lung disease. The repeated thorax CT showed lymph node packs. Also seen were a round lesion of $47 \times 37 \times 30 \mathrm{~mm}$ in the right lung and bilateral parenchymal micronodular lesions. We diagnosed the patient as stage two juvenile sarcoidosis with constitutional symptoms, mediastinal lymphadenomegaly, nodular densities of the lungs, uveitis, arthritis, and hypercalciuria. The initial treatment was prednisone and methotrexate. After treatment his pains regressed, his sedimentation level decreased, and he gained weight. In the first week of the treatment myoclonic jerks occurred but electroencephalography, cranial magnetic resonance (MR) imaging, and MR angiography were normal. The etiology of myoclonus remained unclear. After two months of fulldose treatment with prednisone and methotrexate, we reduced the dosage of prednisone. His pains increased and he developed a new cervical lymph node. With suspicion of a new malignancy, we repeated the PET-CT. Cervical, supraclavicular, mediastinal, and right lung middle lobe hypermetabolic lymph 
nodes were detected. Standardized uptake values (SUV) increased considerably in comparison to the FDG screening which had been done four months before; SUV values ranged between 11.4 and $20 .^{5}$ A cervical lymph node biopsy was done. Four months after the second biopsy, pathologic investigation revealed lymphocyte-rich classical Hodgkin lymphoma (Figure 3).

The ABVD (adriamycin, bleomycin, vinblastine, and decarbazine) chemotherapy regimen was started for stage 2 Hodgkin's lymphoma. With the beginning of the treatment the patient's general health status improved. But after the end of chemotherapy, the nodular lesion on his right lung persisted so a biopsy was taken and it was compatible with a hyalinisated nodule. The treatment continued with local radiotherapy involving cervical and mediastinal regions. After chemotherapy and radiotherapy, the control FDG PET-CT revealed no lesions. At the time of writing, he has had no pain or novel lymph nodes during the past two years.

\section{Discussion}

The patient who presented with constitutional symptoms, mediastinal and cervical lymphadenopathy was diagnosed with Hodgkin's lymphoma only on third biopsy His symptoms mimicking sarcoidosis and tuberculosis, in fact, complicated the diagnostic process.

He had weight loss, malaise, low grade fever, severe ache, cervical and mediastinal lymph nodes on invitation and referred to our clinic with suspicion of tuberculosis (TB). Lymph node TB is a common cause of lymphadenopathy in areas in which TB is endemic as in Turkey. Mediastinal involvement constitutes $44 \%$ of lymph node tuberculosis with pulmonary manifestations. ${ }^{6}$ However, in our case, tuberculosis bacilli were absent in his lymph nodes and gastric aspirates. The skin test was non-reactive, conventional cultures were negative and pathology specimens showed no evidence of caseous necrosis. Diagnosis of tuberculosis was excluded.

Juvenile sarcoidosis is a rare, chronic, multisystem, granulomatous disease. ${ }^{7,8}$ This form of sarcoidosis is characterized by systemic features such as pulmonary and lymph node involvement. At the first referral, $60-98 \%$ of patients presents with malaise, fever, and weight loss. Uveitis, arthritis and erythema nodosum were also reported.$^{8,9}$ No specific laboratory test exists for sarcoidosis and elevation of ACE is not consistent. ${ }^{10}$ Our patient had weight loss, malaise, low grade fever, seizures, arthritis, uveitis, erythema nodosum, mediastinal lymphadenomegaly, hypercalciuria, high ACE level, and a low parathyroid level con- sistent with high 1,25 dihydroxy-vitamin D. The diagnosis of sarcoidosis is greatly supported by showing noncaseating epitheloid and giant cell granulomas in pathologic specimens. But all of the biopsy specimens were negative for sarcoidosis in our case. He had taken prednisone pills and we thought that this may had masked the pathognomonic features of sarcoidosis in biopsy. Because of the clinical and laboratory findings similar to sarcoidosis, and the absence of lymphoma in biopsy specimens, he had been treated as sarcoidosis for four months.

Lymphoma, a systemic disease in which the mediastinal lymph nodes are often involved, can be easily confused with mediastinal tuberculous and sarcoidosis.111 Immunosuppressive drugs like methotrexate can be carcinogenic or could facilitate activation of procarcinogenic viruses such as the EBV. ${ }^{3}$ Our patient had also methotrexate treatment and his EBV DNA value was highly positive. He developed a new cervical lymph node during steroid treatment and his SUV max values increased to malignant levels within four months. Both sarcoid and lymphomas are FDG avid. While SUV values greater than 13 are counted as a malignant formation, sarcoidosis



Figure 1. Patient's disease timeline.
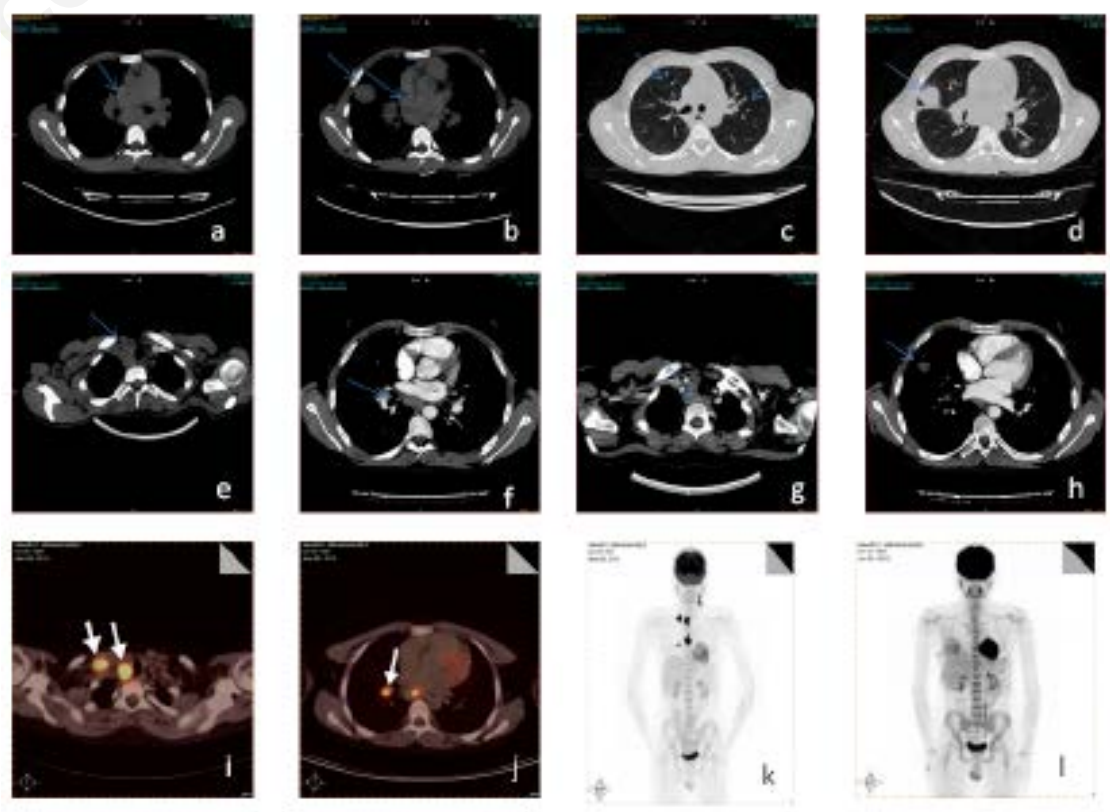

Figure 2. Computed tomography and positron emission tomography-computed tomography imaging. 

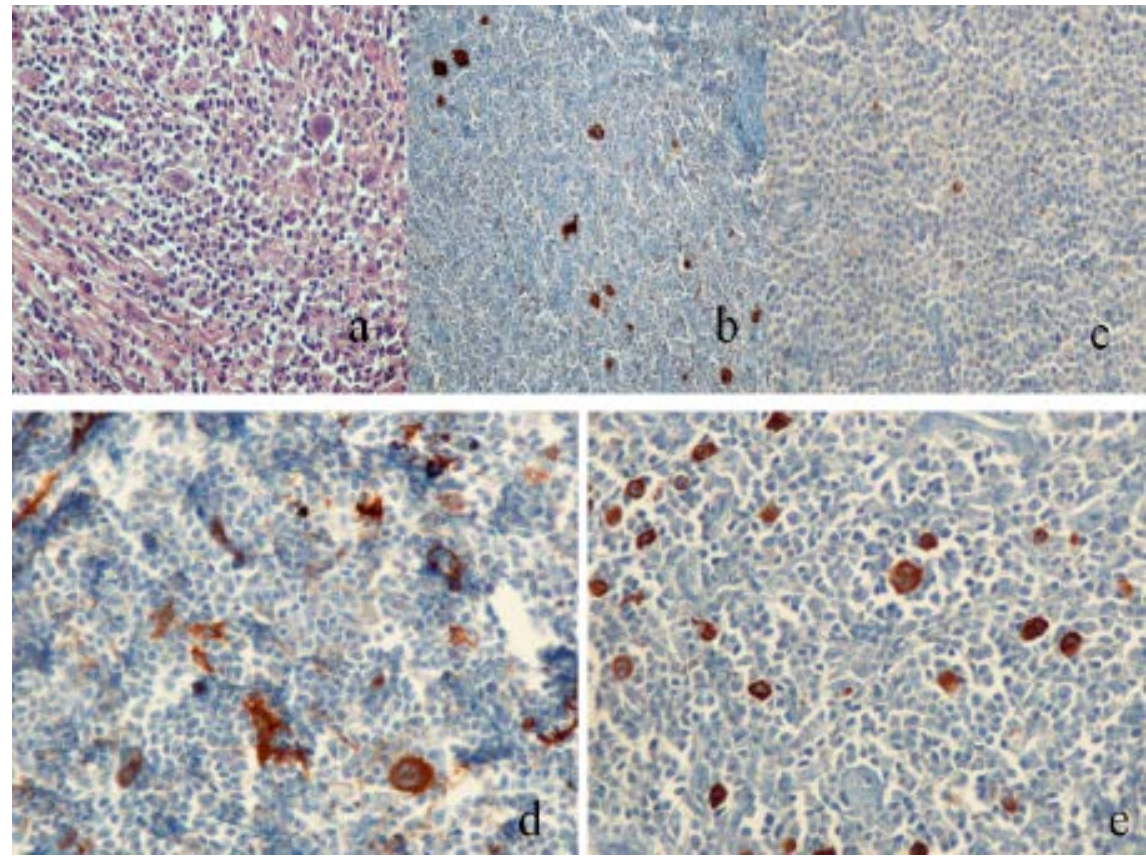

Figure 3. Microscopic examinations.

has a SUV max value of 2.4 to 10.9 $(4.6 \pm 3.2) .{ }^{12,13}$ In our case, the first SUV values were below 13 , which could indicate sarcoidosis, but his SUV values increased up to 20.5 when he was diagnosed with Hodgkin's lymphoma. The first two biopsies were negative for malignancy and the third proved positive for Hodgkin's lymphoma.

\section{Conclusions}

In conclusion, the definitive diagnosis in our case was HL and could be done after eight months of his initial symptoms. The diagnosis of lymphoma may not be easy because of overlapping clinical and histopathological findings with tuberculosis and sarcoidosis. Patients with mediastinal and cervical lymphadenopathy and constitutional symptoms should be investigated extensively and repeated biopsies should be done in high risk patients to establish accurate diagnosis.

\section{References}

1. Gobbi PG, Ferreri A, Ponzoni M, et al. Hodgkin lymphoma. Crit Rev Oncol Hematol 2013;85:216-37.

2. Hodgson DC, Hudson MM, Constine LS. Pediatric hodgkin lymphoma: maximizing efficacy and minimizing toxicity. Semin Radiat Oncol 2007;17:230-42.
3. Munoz N, Davidson RJ, Witthoff B, et al. Infectious mononucleosis and Hodgkin's disease. Int J Cancer 1978;22:10-3.

4. Hyalgrim H. On the aetiology of Hodgkin lymphoma. Dan Med J 2012;59:B4485.

5. Moradi G, Naieni KH, Rashidien A, et al. Evaluation of tuberculosis situation in economic cooperation countries in 2009; achievement and gaps toward millenium development goals. Int J Prev Med 2012;3: 77-83.

6. Tatar D, Senol G, Alptekin S, et al. Assessment of lymph node tuberculosis in two provinces in Turkey. Jpn J Infect Dis 2011;64:316-21.

7. Lazarus A. Sarcoidosis: epidemiology, etiology, pathogenesis, and genetics. Dis Mon 2009;55:649-60.

8. Fretzayas A, Moustaki M, Vougiouka 0. The puzzling spectrum and course of juvenile sarcoidosis. World J Pediatr 2011;7: 103-10.

9. Rose CD, Wouters CH. Pediatric sacoidosis. In: Cassidy JT, Pretty R, Laxer R, Lindsley C, eds. Textbook of pediatric rheumatology. 6th ed. Philadelphia; 2011. pp 544-551.

10. Shetty AK, Gedalia A. Childhood sarcoidosis: a rare but fascinating disorder. Pediatr Rheumatol Online J 2008;23 6-16.

11. Gupta D, Agarwal R, Aggarwal AN, et al. Sarcoidosis and tuberculosis: the same disease with different manifestations or similar manifestations of different disorders. Curr Opin Pulm Med 2012;18:506-16.

12. Schöder H, Noy A, Gönen M, et al. Intensity of 18fluorodeoxyglucose uptake in positron emission tomography distinguishes between indolent and aggressive non-Hodgkin's lymphomas. J Clin Oncol 2005;23:4643-51.

13. Kumar A, Dutta R, Kannan U, et al. Evaluation of mediastinal lymph nodes using 18F-FDG PET CT scan and its histopathologic correlation. Ann Thorac Med 2011;6:11-6. 